Spin-orbit interaction and asymmetry effects on Kondo ridges at finite magnetic field

Grap, Stephan; Andergassen, Sabine; Paaske, Jens; Meden, Volker

Published in:

Physical Review B

DOI:

10.1103/PhysRevB.83.115115

Publication date:

2011

Document version

Early version, also known as pre-print

Citation for published version (APA):

Grap, S., Andergassen, S., Paaske, J., \& Meden, V. (2011). Spin-orbit interaction and asymmetry effects on Kondo ridges at finite magnetic field. Physical Review B, 83(11), 115115.

https://doi.org/10.1103/PhysRevB.83.115115 


\title{
Spin-orbit interaction and asymmetry effects on Kondo ridges at finite magnetic field
}

\author{
S. Grap, ${ }^{1}$ S. Andergassen, ${ }^{1}$ J. Paaske, ${ }^{2}$ and V. Meden ${ }^{1}$ \\ ${ }^{1}$ Institut für Theorie der Statistischen Physik, RWTH Aachen, D-52056 Aachen, Germany and \\ Jülich Aachen Research Alliance -Fundamentals of Future Information Technology \\ ${ }^{2}$ Nano-Science Center, Niels Bohr Institute, Universitetsparken 5, DK-2100 Copenhagen, Denmark \\ (Received 26 November 2010; revised manuscript received 25 January 2011; published 11 March 2011)
}

\begin{abstract}
We study electron transport through a serial double quantum dot with Rashba spin-orbit interaction (SOI) and Zeeman field of amplitude $B$ in the presence of local Coulomb repulsion. The linear conductance as a function of a gate voltage $V_{g}$ equally shifting the levels on both dots shows two $B=0$ Kondo ridges, which are robust against SOI as time-reversal symmetry is preserved. As a result of the crossing of a spin-up and a spin-down level at vanishing SOI, two additional Kondo plateaus appear at finite $B$. They are not protected by symmetry and rapidly vanish if the SOI is turned on. Left-right asymmetric level-lead couplings and detuned on-site energies lead to a simultaneous breaking of left-right and bonding-antibonding state symmetry. In this case, the finite- $B$ Kondo ridges in the $V_{g}-B$ plane are bent with respect to the $V_{g}$ axis. For the Kondo ridge to develop, different level renormalizations must be compensated by adjusting $B$.
\end{abstract}

DOI: 10.1103/PhysRevB.83.115115

PACS number(s): 05.60.Gg, 71.10.-w, 73.63.Kv, 76.20.+q

\section{INTRODUCTION}

In linear response transport through quantum dots, the spin Kondo effect shows up as a plateau in the linear conductance $G$ when varying the level positions by an external gate voltage $V_{g}$, often referred to as a Kondo ridge. ${ }^{1-7}$ The width of the Kondo ridge is set by the local Coulomb interaction $U$ on the dot, which, in the Kondo regime, exceeds the level-lead hybridization $\Gamma$. The latter determines the width of the Lorentzian resonance in $G\left(V_{g}\right)$ at $U=0$. The breaking of the two-fold Kramers degeneracy by a local Zeeman field of amplitude $B$ destroys the Kondo ridge; along the $V_{g}$ axis, the conductance plateau is split up into two Lorentzian resonances. In contrast, spin-orbit interaction (SOI), although breaking spin-rotational symmetry by designating a certain (spin) direction, does not destroy the Kondo effect. ${ }^{8-10}$ In the presence of SOI, spin is no longer a good quantum number, but a Kramers doublet remains as time-reversal symmetry is conserved. This leads to a Kondo effect in the presence of a local interaction, provided the gate voltage is tuned such that the dot is filled (on average) by an odd number of electrons (dominant spin fluctuations).

In multilevel dots, increasing $B$ might lead to energetically degenerate states (level crossings) resulting from different orbitals. If one is a spin-up state and one is a spin-down state, and the gate voltage is tuned such that an electron fluctuates between these states, one might expect the emergence of a spin Kondo effect at finite magnetic field. ${ }^{11-13}$ If the orbital quantum number is conserved in the leads in such systems, additional orbital Kondo effects ${ }^{14}$ and combinations of spin and orbital Kondo effects ${ }^{15}$ may appear. Here we consider a setup where the orbital quantum number does not arise in the leads and we thus concentrate on spin Kondo effects. In contrast to the standard $B=0$ Kondo effect, the one appearing at finite $B$ is not protected by time-reversal symmetry and we show that it is destroyed in the presence of a finite SOI.

Here we study a serial double quantum dot, each having a single spin-degenerate level (at vanishing magnetic field) described by a tight-binding model with two lattice sites coupled by electron hopping of amplitude $t$ and connected to two semi-infinite noninteracting (Fermi liquid) leads via tunnel couplings of strength $\Gamma_{L}$ and $\Gamma_{R}$. The on-site energies of the two levels are given by $\epsilon_{1 / 2}=V_{g} \pm \delta$. The Rashba SOI identifies the $z$ direction of the spin space and is modeled as an imaginary electron hopping with spin-dependent sign between the two lattice sites. ${ }^{9,16-18}$ Here we exclusively consider the coupling of a magnetic field to the spin degree of freedom (Zeeman term) and neglect its effect on the orbital motion. The relevant component of the Zeeman field perpendicular to the SOI defines (without loss of generality) the $x$ direction. The local Coulomb repulsion is modeled as an on-site $U$, as well as a nearest-neighbor $U^{\prime}$ repulsion, and treated within an approximate static functional-renormalization group (fRG) approach. ${ }^{19}$ Our model is sketched in Fig. 1.

In the absence of SOI, a finite- $B$ Kondo effect will lead to a Kondo ridge. This conductance plateau can be detected if $G$ is computed (or measured) as a function of $V_{g}$ and $B$. We show that the finite- $B$ conductance plateau develops on a line parallel to the $V_{g}$ axis, the only exception being the case with broken left-right and bonding-antibonding state symmetry realized for $\Gamma_{L} \neq \Gamma_{R}$ and $\delta \neq 0$. In this case, the finite- $B$ Kondo ridges are bent with respect to the $V_{g}$ axis; to follow the conductance maximum when changing $V_{g}$, one has to adjust $B$ to compensate for the asymmetryinduced level renormalization. As the two symmetries are generically broken in experimental systems, our results are important for the understanding of measurements of the linear conductance of multilevel quantum dots as a function of $V_{G}$ and $B$.

This paper is organized as follows. In the next section, we introduce our double-dot model. In Sec. III details of the approximate fRG treatment of the Coulomb interaction specific to the present model are discussed. For later reference, we also give a brief account of the appearance of the Kondo ridge for a single dot within our approximation scheme. In Sec. IV we discuss our results. We first describe the effects of the SOI on the spectrum of the noninteracting isolated double dot. Next we present our results for the linear conductance $G\left(V_{g}, B\right)$ considering the entire parameter space. Our work is summarized in Sec. V. 


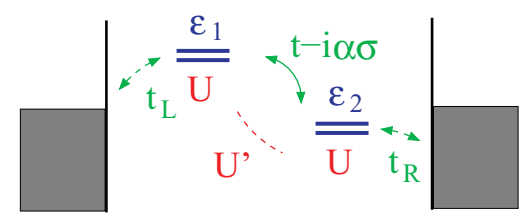

FIG. 1. (Color online) The considered setup consists of a serial double quantum dot with energies $\epsilon_{1 / 2}=V_{g} \pm \delta$ coupled by a hopping amplitude $t$ and a SOI of strength $\alpha$. The levels are split by an external Zeeman field $B$. The local Coulomb interaction is $U$ and the interaction between electrons on the two sites is $U^{\prime}$. The system is coupled to noninteracting leads by hopping amplitudes $t_{L / R}$.

\section{MODEL}

Our multilevel quantum-dot model is realized by a serial double dot, each having a single spin-degenerate level (at $B=$ 0 ) as sketched in Fig. 1. The Hamiltonian of the isolated dot contains several terms,

$$
H_{\text {dot }}=H_{0}+H_{\text {SOI }}+H_{Z}+H_{\text {int }} .
$$

The free part,

$$
H_{0}=\sum_{\sigma}\left[\sum_{j=1,2} \epsilon_{j} d_{j, \sigma}^{\dagger} d_{j, \sigma}-t\left(d_{2, \sigma}^{\dagger} d_{1, \sigma}+\text { H.c. }\right)\right],
$$

with $d_{j, \sigma}^{\dagger}$ being the creation operator of an electron on the dot site $j=1,2$ (Wannier states) of spin $\sigma$, contains the conventional hopping $t>0$, and the on-site energies

$$
\epsilon_{1 / 2}=V_{g} \pm \delta
$$

which can be tuned by an external gate voltage $V_{g}$. The difference of the on-site energies is parametrized by the level-splitting $\delta$. The effect of SOI is taken into account by an imaginary hopping amplitude of spin-dependent sign. It is the lattice realization of a Rashba SOI resulting from spatial confinement. ${ }^{9,16-18}$ The Rashba hopping term with amplitude $\alpha>0$ reads

$$
H_{\mathrm{SOI}}=\alpha \sum_{\sigma, \sigma^{\prime}}\left[d_{2, \sigma}^{\dagger}\left(i \sigma_{z}\right)_{\sigma, \sigma^{\prime}} d_{1, \sigma^{\prime}}+\text { H.c. }\right],
$$

with the third Pauli matrix $\sigma_{z}$. Choosing the $z$ direction in spin space for the direction of the SOI breaks the spin-rotational invariance. The Zeeman field can have one component parallel to the SOI (that is, in $z$ direction) and one perpendicular to it. For this, here we choose (without loss of generality) the $x$ direction such that the (local) Zeeman term reads

$H_{Z}=B \sum_{\sigma, \sigma^{\prime}} \sum_{j=1,2}\left[d_{j, \sigma}^{\dagger}\left(\sigma_{z}\right)_{\sigma, \sigma^{\prime}} d_{j, \sigma^{\prime}} \sin \theta+d_{j, \sigma}^{\dagger}\left(\sigma_{x}\right)_{\sigma, \sigma^{\prime}} d_{j, \sigma^{\prime}} \cos \theta\right]$.
For $\theta= \pm \pi / 2$, the SOI and the $B$ field are antiparallel. In this case, the conventional hopping and the SOI can be combined to an effective hopping and the SOI does not have a specific effect. In particular, it does not destroy the finite- $B$ Kondo ridges (see Sec. IV A). The local Coulomb interaction is included by

$$
H_{\text {int }}=U \sum_{j=1,2}\left(n_{j, \uparrow}-\frac{1}{2}\right)\left(n_{j, \downarrow}-\frac{1}{2}\right)+U^{\prime}\left(n_{1}-1\right)\left(n_{2}-1\right)
$$

for the local $U>0$ and nearest-neighbor $U^{\prime}>0$ interactions, respectively, with $n_{j, \sigma}=d_{j, \sigma}^{\dagger} d_{j, \sigma}$ and $n_{j}=\sum_{\sigma} n_{j, \sigma}$. By subtracting $1 / 2$ from $n_{j, \sigma}$ in the definition of $H_{\text {int }}$, the point $V_{g}=0$ corresponds to half filling of the double dot, even in the presence of Coulomb repulsion.

The dot Hamiltonian is supplemented by a term describing two semi-infinite noninteracting leads, which we model here as one-dimensional tight-binding chains,

$$
H_{\text {lead }}=-\tau \sum_{\beta=L, R} \sum_{j=0}^{\infty} \sum_{\sigma}\left[c_{\beta, j+1, \sigma}^{\dagger} c_{\beta, j, \sigma}+\text { H.c. }\right],
$$

with lead operators $c_{\beta, j+1, \sigma}^{(\dagger)}$ and equal bandwidth $4 \tau$. In the following, we choose $\tau$ as the unit of energy $\tau=1$. The dotlead couplings are given by a tunnel Hamiltonian

$$
H_{\text {coup }}=\sum_{\sigma}\left(t_{L} d_{1, \sigma}^{\dagger} c_{L, 1, \sigma}+t_{R} c_{R, 1, \sigma}^{\dagger} d_{2, \sigma}+\text { H.c. }\right),
$$

with tunnel barriers set by $t_{L / R}$. We consider here the so-called wideband limit (see, e.g., Ref. 19) in which the tunnel barriers only enter in combination with the local lead density of states (at lattice site 1) evaluated at the chemical potential by $\Gamma_{L / R}=$ $\pi t_{L / R}^{2} \rho_{\text {leads. }}$.

\section{METHOD: FUNCTIONAL RENORMALIZATION GROUP}

\section{A. Flow equation for the self-energy}

We briefly review the applied approximation scheme, which is based on the fRG method, ${ }^{20}$ focusing on aspects specific to the present model. A detailed description of the implementation for quantum-dot systems in the absence of SOI is provided in Ref. 19. Recent extensions including SOI involve inhomogeneous quantum wires. ${ }^{18}$

The starting point of the fRG scheme is the bare $(U=$ $\left.U^{\prime}=0\right)$ Matsubara frequency propagator $\mathcal{G}_{0}$ of the double dot. The leads are projected onto the dot sites and enter via the hybridization $\Gamma_{L / R} \cdot{ }^{19}$ In the basis

$$
\{|1, \uparrow\rangle,|1, \downarrow\rangle,|2, \uparrow\rangle,|2, \downarrow\rangle\}
$$

of single-particle states, its inverse reads

$$
\begin{aligned}
& \mathcal{G}_{0}^{-1}(i \omega) \\
& =\left(\begin{array}{cccc}
i \omega-\epsilon_{1}-B \sin \theta+i \Gamma_{L}(\omega) & -B \cos \theta & t-i \alpha & 0 \\
-B \cos \theta & i \omega-\epsilon_{1}+B \sin \theta+i \Gamma_{L}(\omega) & 0 & t+i \alpha \\
t+i \alpha & 0 & i \omega-\epsilon_{2}-B \sin \theta+i \Gamma_{R}(\omega) & -B \cos \theta \\
0 & t-i \alpha & -B \cos \theta & i \omega-\epsilon_{2}+B \sin \theta+i \Gamma_{R}(\omega)
\end{array}\right)
\end{aligned}
$$


with $\Gamma_{L / R}(\omega)=\Gamma_{L / R} \operatorname{sgn}(\omega)$. This propagator is replaced by one in which low-energy degrees of freedom below a cutoff $\Lambda$ are suppressed,

$$
\mathcal{G}_{0}^{\Lambda}(i \omega)=\Theta(|\omega|-\Lambda) \mathcal{G}_{0}(i \omega) .
$$

The cutoff $\Lambda$ is later sent from $\infty$ down to 0 . Inserting $\mathcal{G}_{0}^{\Lambda}$ in the generating functional of the one-particle irreducible vertex functions, an infinite hierarchy of coupled differential equations is obtained by differentiating the generating functional with respect to $\Lambda$ and expanding it in powers of the external fields. Practical implementations require a truncation of the flow-equation hierarchy.

We restrict the present analysis to the first order in the hierarchy and only consider the flow of the one-particle vertex, that is, the self-energy. It was previously discussed analytically $^{19,21}$ that for a single dot, the resulting flow equations capture the appearance of a Kondo ridge in $G\left(V_{g}\right)$ of width $\sim 1.5 U$. The accuracy can be improved by including the flow of the static part of the two-particle vertex (effective interaction), which gives a Kondo plateau of width $\sim U$ in good agreement with the exact result. ${ }^{19,21}$ Here we are not interested in such quantitative improvements and instead keep the bare vertex. This has the advantage that the resulting flow equations for the matrix elements of the self-energy have a simple structure, which not only allows for a fast numerical solution but provides the opportunity to gain analytical insights.

The flow equation for the self-energy reads ${ }^{19}$

$$
\frac{\partial}{\partial \Lambda} \Sigma_{a^{\prime}, a}^{\Lambda}=-\frac{1}{2 \pi} \sum_{\omega= \pm \Lambda} \sum_{b, b^{\prime}} e^{i \omega 0^{+}} \mathcal{G}_{b, b^{\prime}}^{\Lambda}(i \omega) \Gamma_{a^{\prime}, b^{\prime} ; a, b},
$$

where the indices $a, a^{\prime} ; b, b^{\prime}$ label the quantum numbers $(j, \sigma), \Gamma_{a^{\prime}, b^{\prime} ; a, b}$ is the two-particle vertex, and the interacting Green function $\mathcal{G}$ is determined by the Dyson equation

$$
\mathcal{G}^{\Lambda}(i \omega)=\left[\mathcal{G}_{0}^{-1}(i \omega)-\Sigma^{\Lambda}\right]^{-1} .
$$

The initial condition for $\Lambda_{0} \rightarrow \infty$ is $\Sigma^{\Lambda_{0}}=0 .{ }^{19}$ In the lowest-order scheme, the two-particle vertex $\Gamma_{a^{\prime}, b^{\prime} ; a, b}$ is given by the bare antisymmetrized interaction. As the bare vertex is frequency independent, the approximate self-energy turns out to be frequency independent. Dynamical contributions are generated only at higher orders. As the latter are important for the conductance at temperatures $T>0$, the current approximation scheme is restricted to $T=0$. The correct temperature dependence of the (single-dot) Kondo ridge is only captured if the flow of a frequency-dependent two-particle vertex, leading to a flowing frequency-dependent self-energy, is kept. ${ }^{22,23}$ Within our approximation, the matrix elements of the self-energy at the end of the flow $\Sigma^{\Lambda=0}$ can be interpreted as interactioninduced renormalizations to the noninteracting model parameters such as the SOI and conventional hopping amplitudes, as well as the on-site energies and the magnetic field. ${ }^{19}$

\section{B. Computation of the linear conductance}

From the self-energy obtained at the end of the flow at $\Lambda=0$, the full propagator, including interaction effects, is determined via the Dyson equation (13). From this, various observables can be computed. ${ }^{19}$ Here we concentrate on the linear conductance. At $T=0$, current-vertex corrections vanish and the Kubo formula for the spin-resolved conductance assumes a generalized Landauer-Büttiker form ${ }^{24}$

$$
G_{\sigma, \sigma^{\prime}}=\frac{e^{2}}{h}\left|\mathcal{T}_{\sigma, \sigma^{\prime}}(0)\right|^{2},
$$

with the effective transmission $\mathcal{T}_{\sigma, \sigma^{\prime}}(0)$ evaluated at the chemical potential. For the present setup, the transmission is given by the $\left(1, \sigma ; 2, \sigma^{\prime}\right)$ matrix element of the full propagator leading to ${ }^{19}$

$$
G_{\sigma, \sigma^{\prime}}=\frac{e^{2}}{h} 4 \Gamma_{L} \Gamma_{R}\left|\mathcal{G}_{1, \sigma ; 2, \sigma^{\prime}}(0)\right|^{2} .
$$

\section{Single-level quantum dot}

Before analyzing the serial double dot, for later reference we briefly discuss the single-level quantum dot within our approximation scheme. ${ }^{19,21}$ In this case, the flow equation for the effective (flowing) level position $V_{\sigma}^{\Lambda}=V_{g}+\sigma B+\Sigma_{\sigma}^{\Lambda}$ is

$$
\frac{d}{d \Lambda} V_{\sigma}^{\Lambda}=\frac{U V_{\bar{\sigma}}^{\Lambda} / \pi}{(\Lambda+\Gamma)^{2}+\left(V_{\bar{\sigma}}^{\Lambda}\right)^{2}},
$$

with initial condition $V_{\sigma}^{\Lambda=\infty}=V_{g}+\sigma B, \bar{\sigma}=-\sigma$, and $\Gamma=$ $\Gamma_{L}+\Gamma_{R}$.

At $B=0$, the level position is spin-independent, $V_{\sigma}^{\Lambda}=V^{\Lambda}$, and the differential equation can be solved analytically. ${ }^{19,21}$ For $U \gg \Gamma$ (in the Kondo regime) and $\left|V_{g}\right| \lesssim$ $0.77 U$, the solution at $\Lambda=0$ is

$$
V=V_{g} \exp \left(-\frac{U}{\pi \Gamma}\right) .
$$

It is this exponential pinning of the renormalized level position to zero (the chemical potential) that leads to the Kondo plateau in the total conductance $G\left(V_{g}, B\right)=G_{\uparrow}\left(V_{g}, B\right)+G_{\downarrow}\left(V_{g}, B\right)$ given by

$$
G\left(V_{g}, B=0\right)=\frac{2 e^{2}}{h} \frac{4 \Gamma_{L} \Gamma_{R}}{\Gamma^{2}} \frac{\Gamma^{2}}{V^{2}+\Gamma^{2}} .
$$

For $U=0$, the level position $V=V_{g}$ is unrenormalized and $G$ reaches its maximum value $G_{\max }=\left(2 e^{2} / h\right) 4 \Gamma_{L} \Gamma_{R} / \Gamma^{2}$ only at the resonance voltage $V_{g}=0$ (Lorentzian resonance of width $\Gamma$ ). For $U>0, V$ is pinned to zero around $V_{g}=0$ for a gate-voltage range of width $\sim 1.5 U$, where the Kondo plateau develops in the conductance $G \simeq G_{\max }$.

We next consider a finite magnetic field $B \neq 0$. The flow equation for the effective $B^{\Lambda}=\left(V_{\uparrow}^{\Lambda}-V_{\downarrow}^{\Lambda}\right) / 2$ reduces to

$$
\frac{d}{d \Lambda} B^{\Lambda}=-\frac{U B^{\Lambda} / \pi}{(\Lambda+\Gamma)^{2}+\left(B^{\Lambda}\right)^{2}},
$$

at $V_{g}=0$. The renormalized magnetic field $B_{\text {ren }}$ at $\Lambda=0$ hence shows the same exponential behavior (with prefactor $B$ instead of $V_{g}$ ) as the renormalized level position given by Eq. (17), except for the reversed sign in the exponent. This leads to a dramatic increase of the renormalized field $B_{\text {ren }}$ compared to the bare one. For $U>0$, the total conductance at $V_{g}=0$,

$$
G\left(V_{g}=0, B\right)=\frac{2 e^{2}}{h} \frac{4 \Gamma_{L} \Gamma_{R}}{\Gamma^{2}} \frac{\Gamma^{2}}{B_{\mathrm{ren}}^{2}+\Gamma^{2}},
$$

in the $B$ direction becomes exponentially (as set by the Kondo scale $)^{1,19,21}$ sharp, instead of Lorentzian-like of width $\Gamma$ for 
$U=0$. In fact, this holds not only at $V_{g}=0$ but for all gate voltages within the $B=0$ conductance plateau. Qualitatively, the Kondo ridge of a single-level dot in the $V_{g}-B$ plane looks similar to the surrounding of one of the $B=0$ Kondo ridges appearing in our double-dot model as shown, e.g., in Fig. 2.

$$
h_{\mathrm{dot}}=\left(\begin{array}{cc}
V_{g}+\delta+B \sin \theta & B \cos \theta \\
B \cos \theta & V_{g}+\delta-B \sin \theta \\
-t-i \alpha & 0 \\
0 & -t+i \alpha
\end{array}\right.
$$

The corresponding eigenvalues are

$$
\begin{aligned}
\lambda & =V_{g} \pm \sqrt{B^{2}+t^{2}+\delta^{2}+\alpha^{2} \pm 2 B \sqrt{t^{2}+\delta^{2}+\alpha^{2} \sin ^{2} \theta}} \\
& =V_{g} \pm \sqrt{\left(B \pm \sqrt{t^{2}+\delta^{2}+\alpha^{2} \sin ^{2} \theta}\right)^{2}+\alpha^{2} \cos ^{2} \theta}
\end{aligned}
$$

The spectrum is invariant under the transformation $B \rightarrow-B$ and symmetric at $V_{g}=0$. A finite on-site energy $\delta>0$ yields an effective hopping $t_{\text {eff }}=\sqrt{t^{2}+\delta^{2}}$. For vanishing SOI $(\alpha=$ $0)$, Eq. (22) reduces to

$$
\lambda=V_{g} \pm\left(t_{\text {eff }} \pm B\right) .
$$

Naturally, the $\theta$ dependence drops out. For $\theta= \pm \pi / 2$, the SOI and the Zeeman field are antiparallel, and $\alpha$ can be absorbed into an effective hopping $t_{\mathrm{eff}}=\sqrt{t^{2}+\delta^{2}+\alpha^{2}}$. The eigenvalues are then of the $\alpha=0$ form given by Eq. (23).

For the appearance of a spin Kondo effect (after turning on $\Gamma_{L / R}$ as well as $U$ and $U^{\prime}$ ), it is necessary that two degenerate levels of opposite spin are located at zero energy (the chemical potential). Zero-energy spin-degenerate levels are obtained at $B=0$ and $V_{g}= \pm t_{\text {eff }}$ (bonding and antibonding states). This will lead to the standard spin Kondo effect related to the presence of a (spin) Kramers doublet when $U$ and $U^{\prime}$ are switched on. By increasing $B$, the spin-up level of the bonding state and the spin-down level of the antibonding state approach each other. For either $\alpha=0$ or $\theta= \pm \pi / 2$, they become degenerate (cross) at zero energy for $B_{c}= \pm t_{\text {eff }}$ and $V_{g}=0$. Besides the two $B=0$ Kondo ridges developing for all

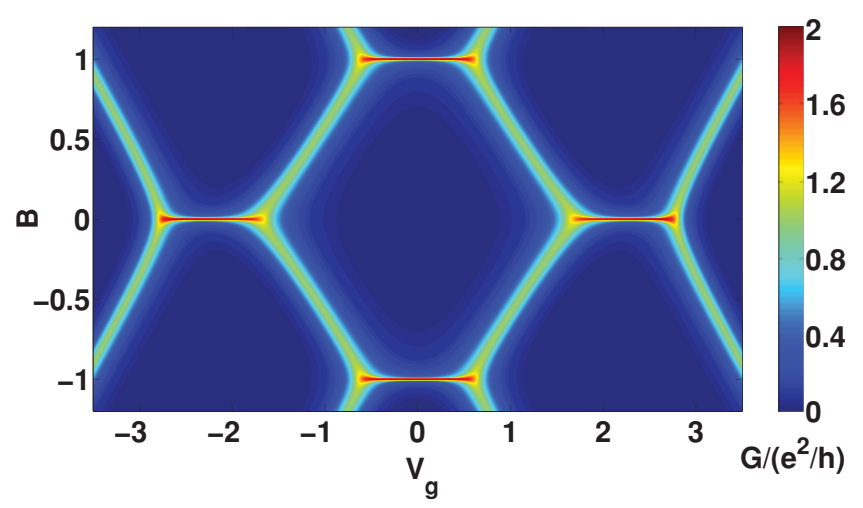

FIG. 2. (Color online) Conductance $G\left(V_{g}, B\right)$ for $t=1, \quad U=$ $U^{\prime}=1, t_{L / R}=0.3$, and $\delta=0$ in the absence of SOI $(\alpha=0)$.

\section{RESULTS}

\section{A. The noninteracting isolated double dot}

For a detailed understanding of the Kondo ridges, it is instructive to first study the noninteracting isolated double dot. In the basis of Eq. (9), the single-particle Hamiltonian $h_{\text {dot }}$ is represented as a complex $4 \times 4$ matrix,

$$
\left.\begin{array}{cc}
-t+i \alpha & 0 \\
0 & -t-i \alpha \\
-\delta+B \sin \theta & B \cos \theta \\
B \cos \theta & V_{g}-\delta-B \sin \theta
\end{array}\right)
$$

dot parameters, for $\alpha=0$ or $\theta=\pi / 2$, one might thus expect the appearance of two finite- $B$ Kondo ridges. Viewed on the basis of the many-body energies of the isolated interacting double dot this situation corresponds to the crossing of a two-particle $S=1, S_{z}=-1$ state with a corresponding $S=1$, $S_{z}=0$ state. The resulting Kondo effect can thus also be referred to as a singlet-triplet one. For $\alpha>0$ and an arbitrary angle $\theta$ between the SOI and the Zeeman field, the finite- $B$ level crossings turn into avoided crossings and no zero-energy degeneracies are possible at finite $B$.

\section{B. Numerical results for the conductance}

We next present results for the linear conductance $G\left(V_{g}, B\right)$ obtained by numerically solving the flow equation (12). For our purposes, it is sufficient to consider only a single set of $U, U^{\prime}$ and $t$. The results depend only quantitatively on the strength of the local Coulomb interaction and the interdot hopping $t$, as long as $U / \Gamma_{L / R}$ and $t / t_{L / R}$ are sufficiently large. We focus here on $U=U^{\prime}=1$ and $t=1$.

\section{Vanishing SOI}

We start our discussion with the case of vanishing SOI. This is trivially realized for $\alpha=0$. As discussed above, for $\theta=\pi / 2$, the SOI can be absorbed into an effective hopping. In this case, the Zeeman field and the SOI are antiparallel and only a single Pauli matrix enters the Hamiltonian. The physics is thus similar to the $\alpha=0$ case.

In Fig. 2 we show $G\left(V_{g}, B\right)$ for a left-right symmetric setup with $t_{L / R}=0.3$ and $\delta=0$, in the absence of SOI $(\alpha=0)$. The conductance shows the expected two pairs of $B=0$ and $V_{g}=0$ Kondo ridges. Due to the renormalization of the interdot hopping, the $B=0$ plateaus are not centered around $V_{g}= \pm t_{\text {eff }}=t=1$, but around renormalized gate voltages. For the present parameters, the renormalization of the interdot hopping and the magnetic field (almost) cancel each other, such that the finite- $B$ ridges are located at $B_{c} \approx \pm t_{\text {eff }}=t=1$. For $U^{\prime}<U, B_{c}$ is renormalized to smaller values. All Kondo plateaus exhibit the same maximal height of $2 e^{2} / h$. This can be understood from transforming the dot-lead coupling given by Eq. (8) into the basis of bonding $(b)$ and antibonding $(\bar{b})$ states of the noninteracting isolated dot. The couplings between the leads and the two states are

$$
\Gamma_{b, L / R}=\frac{\sqrt{t^{2}+\delta^{2}} \mp \delta}{2 \sqrt{t^{2}+\delta^{2}}} \Gamma_{L / R},
$$






FIG. 3. (Color online) Gate-voltage dependence of the conductance $G$ and dot occupation $\left\langle n_{1}+n_{2}\right\rangle$ for different values of $B$, and the same parameters as in Fig. 2.

and $\Gamma_{\bar{b}, L / R}=\Gamma_{b, R / L} t_{L / R}^{2} / t_{R / L}^{2}$. For the considered left-right symmetric case and $\delta=0$, the bonding and antibonding states have the same total coupling $\Gamma_{b / \bar{b}}=\Gamma_{b / \bar{b}, L}+\Gamma_{b / \bar{b}, R}$, and the couplings are left-right symmetric, implying unitary conductance. Increasing $|B|$ from $B=0$, the Kondo ridges are suppressed on the exponential Kondo scale and resonance peaks of height $e^{2} / h$ and width $\Gamma$ develop. The position of the resonance peaks varies linearly with $V_{g}$. Eventually the peaks corresponding to the spin-up level of the bonding state and the spin-down level of the antibonding state merge with the finite- $B$ Kondo ridges. Figure 3 shows $G\left(V_{g}\right)$ at fixed, different $B>0$ to further exemplify this. The shoulders appearing in the bonding (antibonding) state of $B=0$ Kondo ridges are linked to the presence of the antibonding (bonding) state. Increasing the Coulomb interaction has the two obvious effects of broadening the Kondo ridges and increasing the distance between the centers of the plateaus; the latter also holds for increasing $t$. In the lower panel of Fig. 3, the respective dot fillings are shown for various values of $B$. For $B=0$, the dot occupation exhibits plateaus at odd fillings. Their width corresponds to the Kondo ridges observed in the conductance. Similarly, for a finite magnetic field $B=1$, the plateau around $V_{g}=0$ in the conductance is reflected in the filling.

We note that $G\left(V_{g}, B\right)$ is symmetric with respect to $B \rightarrow$ $-B$ [see the eigenvalues given by Eq. (22)] and $V_{g} \rightarrow-V_{g}$. While the former symmetry is given by the Hamiltonian and holds for all parameter sets, the latter is specific to the parameters chosen here $\left(t_{L}=t_{R}\right.$ and $\left.\delta=0\right)$. The conductance remains symmetric under $V_{g} \rightarrow-V_{g}$ if at least either $\delta=0$ or $t_{L}=t_{R}$ holds. In these cases, the four Kondo ridges no longer reach the unitary value $2 e^{2} / h$, but exhibit an equally reduced conductance plateau as the bonding and antibonding states are coupled with the same asymmetry and the same total coupling to the leads.

The most interesting situation arises if the left-right symmetry and the bonding-antibonding state symmetries are simultaneously broken. This is achieved for $t_{L} \neq t_{R}$ and $\delta \neq 0$. In this case, the $V_{g} \rightarrow-V_{g}$ symmetry of $G\left(V_{g}, B\right)$ is broken, as shown in Fig. 4. For our parameters, the couplings of the antibonding state have a strong left-right asymmetry leading to a $B=0$ Kondo plateau with significantly reduced conductance (around $V_{g}=2.2$ ). The bonding state has a weaker asymmetry,

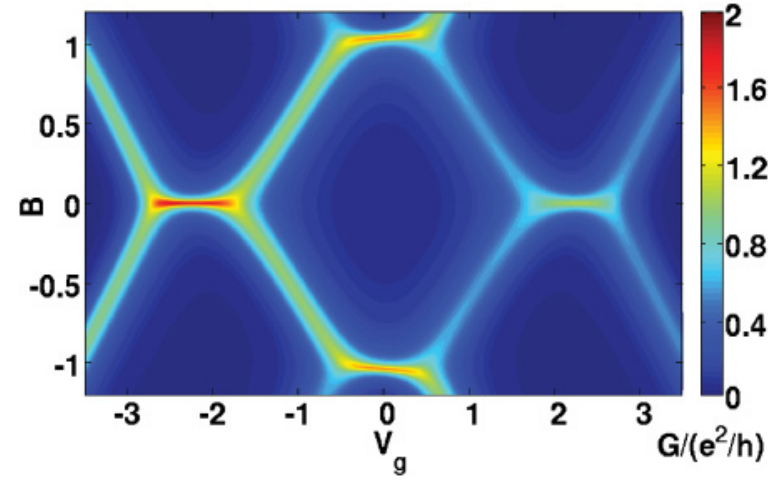

FIG. 4. (Color online) Conductance $G\left(V_{g}, B\right)$ for $t=1, \quad U=$ $U^{\prime}=1$, and $\alpha=0$ with asymmetric couplings to the leads $t_{L}=$ $0.3, t_{R}=0.5$, and finite level-splitting $\delta=-0.3$.

such that the $B=0$ Kondo ridge centered around $V_{g}=-2.2$ almost reaches the unitary conductance. The total coupling $\Gamma_{\bar{b}}$ is larger than $\Gamma_{b}$. With the breaking of the $V_{g} \rightarrow-V_{g}$ symmetry, manifest already from the comparison of the two $B=0$ Kondo ridges, the finite- $B$ Kondo ridges (centered around $V_{g}=0$ ) are no longer necessarily parallel to the $V_{g}$ axis. In fact they are bent with respect to this axis, as becomes apparent from Fig. 4. It turns out that the direction of bending is always away from the state with stronger total level-lead coupling. For our model, this is the state with larger left-right asymmetry. This bending cannot be predicted considering the isolated double dot, even by including the Coulomb interaction as it results from a level renormalization associated with the dot-lead couplings. Related level renormalizations are discussed in Refs. 25 and 26. In experiments on multilevel quantum dots, left-right symmetry is difficult to realize and the states at different energies will have different level-lead couplings. Therefore finite- $B$ spin Kondo ridges appearing in measurements are expected to be generically bent with respect to the $B=0$ ones. This result is relevant for the understanding of finite- $B$ Kondo ridges observed in conductance measurements on multilevel carbon nanotube quantum dots. ${ }^{27}$

\section{Effect of the SOI}

For the discussion of the effect of the SOI on the Kondo ridges, we focus on the symmetric case with $t_{L}=t_{R}$ and $\delta=0$.

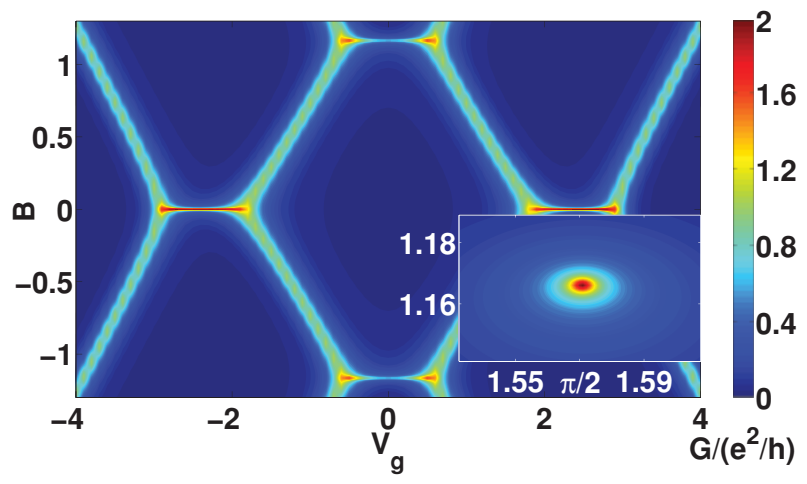

FIG. 5. (Color online) Conductance $G\left(V_{g}, B\right)$ for $t=1, \quad U=$ $U^{\prime}=1, t_{L / R}=0.3$, and $\delta=0$ with $\theta=1.56$ and finite SOI $\alpha=0.6$. Inset: Conductance $G(\theta, B)$ at $V_{g}=0$. 
Figure 5 shows $G\left(V_{g}, B\right)$ for $\alpha=0.6$ and an angle $\theta=1.56$ close to the parallel configuration with $\theta=\pi / 2$. Thus the effective SOI given by the component perpendicular to the direction of the Zeeman field is small. For the noninteracting single-particle levels of the isolated double dot, this implies a small minimal distance between the levels avoiding the crossing at finite $B$. Therefore remnants of the finite- $B$ Kondo ridges are still observable. The inset shows $G$ at $V_{g}=0$ as a function of $\theta$ and $B$. Increasing the SOI component perpendicular to the direction of the Zeeman field by deviating from $\theta=\pi / 2$ obviously destroys the finite- $B$ Kondo effect, as expected. This provides a way to probe the presence of SOI in multilevel dots: after observing finite- $B$ spin Kondo ridges, one can probe their robustness by changing the direction of the magnetic field. Remarkably, the SOI introduces an intriguing angular dependence even for a magnetic field coupling exclusively to the spin degree of freedom. ${ }^{10}$ From the angular dependence of the gap opening in the single-particle energy spectrum the strength of the SOI can be extracted by spectroscopic measurements.

\section{Analytical insights}

We next provide analytical insights to our findings in the absence of SOI by analyzing the fRG flow equation (12). To simplify the analysis, we focus on the case of purely local Coulomb interactions with $U^{\prime}=0$. Due to the absence of Fock terms, $t$ remains unrenormalized, but this only yields quantitative changes compared to the results shown in the last subsection.

In the absence of SOI, $\theta$ does not play any role and can be chosen arbitrarily. For $\theta= \pm \pi / 2$, the matrix (10) is block diagonal and we can restrict the analysis to a single spin sector. Introducing $V_{j \sigma}^{\Lambda}=\epsilon_{j}+\sigma B+\Sigma_{j \sigma}^{\Lambda}$, the full propagator including the self-energy reads

$$
\mathcal{G}_{\sigma}^{\Lambda}(i \omega)=\frac{1}{D_{\sigma}^{\Lambda}(i \omega)}\left(\begin{array}{cc}
i \omega+i \Gamma_{R} \operatorname{sgn}(\omega)-V_{2 \sigma}^{\Lambda} & -t \\
-t & i \omega+i \Gamma_{L} \operatorname{sgn}(\omega)-V_{1 \sigma}^{\Lambda}
\end{array}\right),
$$

with the determinant

$$
D_{\sigma}^{\Lambda}(i \omega)=\left[i \omega+i \Gamma_{R} \operatorname{sgn}(\omega)-V_{2 \sigma}^{\Lambda}\right]\left[i \omega+i \Gamma_{L} \operatorname{sgn}(\omega)-V_{1 \sigma}^{\Lambda}\right]-t^{2}
$$

The zeros of $D_{\sigma}^{\Lambda=0}(0)$ for $\Gamma_{L / R}=0$ determine the zero-energy levels. For degenerate levels, these are responsible for the development of the Kondo ridges in the conductance.

Inserting the above expression in Eq. (12), the flow equations for the local potential are

$$
\frac{d}{d \Lambda} V_{1 \sigma}^{\Lambda}=\frac{U}{\pi} \frac{\left(V_{1 \bar{\sigma}}^{\Lambda} V_{2 \bar{\sigma}}^{\Lambda}-t^{2}\right) V_{2 \bar{\sigma}}^{\Lambda}+\left(\Lambda+\Gamma_{R}\right)^{2} V_{1 \bar{\sigma}}^{\Lambda}}{\left[V_{1 \bar{\sigma}}^{\Lambda} V_{2 \bar{\sigma}}^{\Lambda}-\left(\Lambda+\Gamma_{L}\right)\left(\Lambda+\Gamma_{R}\right)-t^{2}\right]^{2}+\left[\left(\Lambda+\Gamma_{R}\right) V_{1 \bar{\sigma}}^{\Lambda}+\left(\Lambda+\Gamma_{L}\right) V_{2 \bar{\sigma}}^{\Lambda}\right]^{2}},
$$

with an analog equation for $V_{2 \sigma}^{\Lambda}$ with $(1 \leftrightarrow 2)$ and $(L \leftrightarrow R)$.

We first analyze the symmetric case for $\delta=0\left(\epsilon_{1}=\epsilon_{2}\right)$ and $\Gamma_{L}=\Gamma_{R}=\Gamma$. The condition for zero-energy levels, $V_{\sigma}^{2}-$ $t^{2}=0$, implies degenerate solutions for either $B=0$ and $V=$ $\pm t$ or $B_{\text {ren }}= \pm t$ and $V=0$, where the renormalized level position or Zeeman field replace the bare ones with respect to the noninteracting case. The flow equation for the local potential (27) reduces to

$$
\begin{aligned}
\frac{d}{d \Lambda} V_{\sigma}^{\Lambda} & =\frac{U V_{\bar{\sigma}}^{\Lambda}}{\pi} \frac{\left(V_{\bar{\sigma}}^{\Lambda}\right)^{2}-t^{2}+(\Lambda+\Gamma)^{2}}{\left[\left(V_{\bar{\sigma}}^{\Lambda}\right)^{2}-t^{2}-(\Lambda+\Gamma)^{2}\right]^{2}+4\left(V_{\bar{\sigma}}^{\Lambda}\right)^{2}(\Lambda+\Gamma)^{2}} \\
& =\frac{U V_{\bar{\sigma}}^{\Lambda}}{\pi} \operatorname{Re} \frac{1}{(\Lambda+\Gamma+i t)^{2}+\left(V_{\bar{\sigma}}^{\Lambda}\right)^{2}},
\end{aligned}
$$

resembling Eq. (16) for the single-level quantum dot, except for the presence of the finite interdot hopping $t$ and a factor $1 / 2$ in the definition of $\Gamma$. At $B=0$, the flow equation for $\tilde{V}^{\Lambda}=$ $V^{\Lambda}-t \operatorname{sgn}\left(V^{\Lambda}\right)$ is characterized by an exponential pinning to zero for $|\tilde{V}| \lesssim 0.77 U$, inducing a splitting of the central plateau for $\tilde{V}$ in two plateaus of half width for $V$ ranging from $V_{g}= \pm t$ to $V_{g}= \pm(t+0.77 U)$. These are shifted to larger values in Fig. 3 as a consequence of the renormalization of $t$. The same substitution around the $B=0$ Kondo ridges $V_{\sigma}^{\Lambda}=t \operatorname{sgn}\left(V^{\Lambda}\right)+\sigma B^{\Lambda}$ leads to an exponential suppression of the renormalized field, as described by Eq. (19) for the single-level dot. A similar behavior in the $V_{g}-B$ plane is found for the finite- $B$ Kondo ridges. Here the renormalization of $B$ leads to a shift of the position. Due to the enhancement of the renormalized magnetic field, the finite- $B$ Kondo ridge develops at a reduced field for which $B_{\text {ren }}= \pm t$. This effect is superposed by the renormalization of $t$ in Fig. 3 and is hardly visible.

We now consider the general asymmetric situation. With $V_{1 / 2 \sigma}^{\Lambda}=\bar{V}_{\sigma}^{\Lambda} \pm \delta$, and introducing the asymmetry parameter $\beta$ for the coupling to the leads $\Gamma_{L / R}=\Gamma \pm \beta$, the flow equation for $\bar{V}_{\sigma}^{\Lambda}$ is

$$
\frac{d}{d \Lambda} \bar{V}_{\sigma}^{\Lambda}=\frac{U}{\pi} \frac{\bar{V}_{\bar{\sigma}}^{\Lambda}\left[\left(\bar{V}_{\bar{\sigma}}^{\Lambda}\right)^{2}-\delta^{2}-t^{2}+\beta^{2}\right]+(\Lambda+\Gamma)\left[(\Lambda+\Gamma) \bar{V}_{\bar{\sigma}}^{\Lambda}-2 \beta \delta\right]}{\left[\left(\bar{V}_{\bar{\sigma}}^{\Lambda}\right)^{2}-\delta^{2}-t^{2}+\beta^{2}-(\Lambda+\Gamma)^{2}\right]^{2}+4\left[(\Lambda+\Gamma) \bar{V}_{\bar{\sigma}}^{\Lambda}-\beta \delta\right]^{2}} .
$$


Analogously, a flow equation for the renormalization of $\delta$ can be derived, which will not be considered in the following as it does not significantly affect the results. For $\delta \neq 0$ and symmetric couplings to the reservoirs $\beta=0\left(\Gamma_{L}=\Gamma_{R}\right)$, the above equation reduces to (28). Kondo plateaus of width $0.77 U$ are hence obtained in correspondence of degenerate energy levels at $\bar{V}= \pm t_{\text {eff }}$ for $B=0$, and at $B_{\text {ren }}= \pm t_{\text {eff }}$ for $V_{g}=0$. For asymmetric couplings to the leads $(\beta \neq 0)$, Eq. (29) in proximity to the degenerate energy levels can be simplified to

$$
\frac{d}{d \Lambda} \bar{V}_{\sigma}^{\Lambda}=\frac{U}{\pi} \frac{\bar{V}_{\bar{\sigma}}^{\Lambda}-2 \beta \delta / \Gamma}{(\Lambda+\Gamma)^{2}+4\left(\bar{V}_{\bar{\sigma}}^{\Lambda}-\beta \delta / \Gamma\right)^{2}},
$$

in the limit of small asymmetric couplings $\beta \ll \Gamma$. The terms proportional to $\beta \delta / \Gamma$ induce a shift in the effective level position responsible for the $V_{g} \rightarrow-V_{g}$ symmetry breaking. For $V_{g} \gtrsim 0$, an effective magnetic field of strength $B_{c}+$ $2 \beta \delta / \Gamma$ is required to compensate for the shift (corresponding to a larger effective field for $\beta \delta>0$ in Fig. 4). This implies that the finite- $B$ Kondo plateaus in the $V_{g}-B$ plane are not parallel to the $B=0$ axis for $\beta \delta \neq 0$; see Fig. 4 .

\section{CONCLUSION}

We studied the linear conductance of a serial quantum dot as a minimal model to describe the influence of the SOI on the spin Kondo effect in the presence of a Zeeman field. ${ }^{28}$ Without SOI, the linear conductance as a function of an applied gate voltage exhibits characteristic Kondo plateaus at finite magnetic field $B$, in addition to the plateaus at $B=0$. Interestingly the finite- $B$ Kondo ridges are bent with respect to the $V_{g}$ axis if the left-right symmetry and the symmetry between the bonding and antibonding states are broken simultaneously. This finding is of importance for the understanding of measurements of the linear conductance of multilevel quantum dots as a function of $V_{G}$ and $B$. In the presence of SOI, the finite- $B$ Kondo ridges disappear; in contrast to the ridges at $B=0$, they are not protected by time-reversal symmetry.

\section{ACKNOWLEDGMENTS}

We are grateful to K. Grove-Rasmussen for inspiration and numerous discussions, and to $\mathrm{C}$. Karrasch for a critical reading of the manuscript. This work was supported by the Deutsche Forschungsgemeinschaft (FOR 912).
${ }^{1}$ A. C. Hewson, The Kondo Problem to Heavy Fermions (Cambridge University Press, Cambridge, 1993).

${ }^{2}$ L. I. Glazman and M. E. Raikh, JETP Lett. 47, 452 (1988).

${ }^{3}$ T. K. Ng and P. A. Lee, Phys. Rev. Lett. 61, 1768 (1988).

${ }^{4}$ D. Goldhaber-Gordon, H. Shtrikman, D. Mahalu, D. AbuschMagder, U. Meirav, and M. A. Kastner, Nature (London) 391, 156 (1998).

${ }^{5}$ S. M. Cronenwett, T. H. Oosterkamp, and L. P. Kouwenhoven, Science 281, 540 (1998).

${ }^{6}$ J. Schmid, J. Weis, K. Eberl, and K. von Klitzing, Physica B 256258, 182 (1998).

${ }^{7}$ W. van der Wiel, S. De Franceschi, T. Fujisawa, J. M. Elzerman, S. Tarucha, and L. P. Kouwenhoven, Science 289, 2105 (2000).

${ }^{8}$ Y. Meir and N. S. Wingreen, Phys. Rev. B 50, 4947 (1994).

${ }^{9}$ J. E. Birkholz, Ph.D. thesis, Universität Göttingen, 2008.

${ }^{10}$ J. Paaske, A. Andersen, and K. Flensberg, Phys. Rev. B 82, 081309(R) (2010).

${ }^{11}$ M. Pustilnik, Y. Avishai, and K. Kikoin, Phys. Rev. Lett. 84, 1756 (2000).

${ }^{12}$ J. Nygård, D. Cobden, and P. E. Lindelof, Nature (London) 408, 342 (2000).

${ }^{13}$ W. Izumida, O. Sakai, and S. Tarucha, Phys. Rev. Lett. 87, 216803 (2001).

${ }^{14}$ D. L. Cox and A. Zawadowski, Adv. Phys. 47, 599 (1998).

${ }^{15}$ L. Borda, G. Zarand, W. Hofstetter, B. I. Halperin, and J. von Delft, Phys. Rev. Lett. 90, 026602 (2003).
${ }^{16} \mathrm{R}$. Winkler, Spin-Orbit Coupling Effects in Two-Dimensional Electron and Hole Systems (Springer, Berlin, 2003).

${ }^{17}$ F. Mireles and G. Kirczenow, Phys. Rev. B 64, 024426 (2001).

${ }^{18}$ J. E. Birkholz and V. Meden, J. Phys. Condens. Matter 20, 085226 (2008); Phys. Rev. B 79, 085420 (2009).

${ }^{19}$ C. Karrasch, T. Enss, and V. Meden, Phys. Rev. B 73, 235337 (2006).

${ }^{20}$ M. Salmhofer and C. Honerkamp, Prog. Theor. Phys. 105, 1 (2001).

${ }^{21}$ S. Andergassen, T. Enss, and V. Meden, Phys. Rev. B 73, 153308 (2006).

${ }^{22}$ C. Karrasch, R. Hedden, R. Peters, Th. Pruschke, K. Schönhammer, and V. Meden, J. Phys. Condens. Matter 20, 345205 (2008); C. Karrasch, V. Meden, and K. Schönhammer, Phys. Rev. B 82, 125114 (2010).

${ }^{23}$ S. G. Jakobs, M. Pletyukhov, and H. Schoeller, Phys. Rev. B 81, 195109 (2010)

${ }^{24}$ A. Oguri, J. Phys. Soc. Jpn. 70, 2666 (2001).

${ }^{25}$ J. V. Holm, H. I. Jørgensen, K. Grove-Rasmussen, J. Paaske, K. Flensberg, and P. E. Lindelof, Phys. Rev. B 77, 161406(R), 2008.

${ }^{26}$ J. R. Hauptmann, J. Paaske, and P. E. Lindelof, Nature Phys. 4, 373 (2008).

${ }^{27} \mathrm{~K}$. Grove-Rasmussen (private communication).

${ }^{28} \mathrm{~A}$ related model specifically designed for the description of carbon nanotube multilevel quantum dots was studied in M. R. Galpin, F. W. Jayatilaka, D. E. Logan, and F. B. Anders, Phys. Rev. 81, 075437 (2010) 